\title{
Spectral Theory For Strongly Continuous Cosine
}

https://doi.org/10.1515/conop-2020-0110

Received August 27, 2020; accepted January 10, 2021

Abstract: Let $(C(t))_{t \in \mathbb{R}}$ be a strongly continuous cosine family and $A$ be its infinitesimal generator. In this work, we prove that, if $C(t)-\cosh \lambda t$ is semi-Fredholm (resp. semi-Browder, Drazin inversible, left essentially Drazin and right essentially Drazin invertible) operator and $\lambda t \notin i \pi \mathbb{Z}$, then $A-\lambda^{2}$ is also. We show by counterexample that the converse is false in general.

Keywords: Cosine, semi-Fredholm, Drazin invertible, semi-Browder, left essentially Drazin invertible, right essentially Drazin invertible.

MSC: 47D09; 47A11

\section{Introduction}

Let $X$ be a complex Banach space, $\mathcal{B}(X)$ denote the algebra of all bounded linear operators on $X$ and $\mathcal{C}(X)$ the set of all linear closed operators from $X$ to $X$. We write $\mathcal{D}(T), \mathcal{R}(T), \mathcal{N}(T), \rho(T), \sigma(T), \sigma_{p}(T), \sigma_{a p}(T)$ and $\sigma_{r}(T)$ respectively for the domain, the range, the kernel, the resolvent, the spectrum, the point spectrum, the approximate point spectrum and residual spectrum of an operator $T \in \mathcal{C}(X)$. The function resolvent of $T \in \mathcal{C}(X)$ is defined for all $\lambda \in \rho(T)$ by $R(\lambda, T)=(\lambda-T)^{-1}$. The ascent $a(T)$, the descent $d(T)$, the essential ascent $a_{e}(T)$ and the essential descent $d_{e}(T)$ of an operator $T \in \mathcal{C}(X)$ are defined respectively by

$$
\begin{gathered}
a(T)=\inf \left\{k \in \mathbb{N}: \mathcal{N}\left(T^{k}\right)=\mathcal{N}\left(T^{k+1}\right)\right\}, \\
d(T)=\inf \left\{k \in \mathbb{N}: \mathcal{R}\left(T^{k}\right)=\mathcal{R}\left(T^{k+1}\right)\right\}, \\
a_{e}(T)=\min \left\{k \in \mathbb{N}: \operatorname{dim} \mathcal{N}\left(T^{k+1}\right) / \mathcal{N}\left(T^{k}\right)<\infty\right\}, \\
d_{e}(T)=\min \left\{k \in \mathbb{N}: \operatorname{dim} \mathcal{R}\left(T^{k}\right) / \mathcal{R}\left(T^{k+1}\right)<\infty\right\},
\end{gathered}
$$

with the convention $\inf (\varnothing)=\infty$, see $([5,13])$. For $T \in \mathcal{C}(X)$, if there is an operator $S \in \mathcal{B}(X)$ with $\mathcal{R}(S) \subseteq \mathcal{D}(T)$ such that $S T S=S, T S x=S T x$ for all $x \in \mathcal{D}(T)$, and $T^{k}(I-T S)=0$ for some $k \in \mathbb{N}$, then $S$ is called a Drazin inverse of $T$. Note that an operator $T \in \mathcal{C}(X)$ has a Drazin inverse if and only if there exists $k \in \mathbb{N}$ such that $a(T)=d(T)=k$ and $X=\mathcal{R}\left(T^{k}\right) \oplus \mathcal{N}\left(T^{k}\right)$, (see [12]). An operator $T \in \mathcal{C}(X)$ is a left essentially Drazin invertible operator if $a_{e}(T)<\infty$ and $\mathcal{R}\left(T^{a_{e}(T)+1}\right)$ is closed. If $d_{e}(T)<\infty$ and $\mathcal{R}\left(T^{a_{e}(T)}\right)$ is closed, then $T$ is called right essentially Drazin invertible. The Drazin invesible, left essentially Drazin and right essentially Drazin invertible spectra are defined by

$$
\begin{gathered}
\sigma_{D}(T)=\{\lambda \in \mathbb{C}: T-\lambda \text { is not Drazin inversible }\}, \\
\sigma_{l d}^{e}(T)=\{\lambda \in \mathbb{C}: T-\lambda \text { is not left essentially Drazin inversible }\}, \\
\sigma_{r d}^{e}(T)=\{\lambda \in \mathbb{C}: T-\lambda \text { is not right essentially Drazin inversible }\} .
\end{gathered}
$$

^Corresponding Author: Hamid Boua: Department of Mathematics, Labo LIABM, FPN, Mohammed First University, PO Box 300, 62702 Selouane, Nador, Morocco ; E-mail: h.boua@ump.ac.ma 
An operator $T \in \mathcal{C}(X)$ is called upper semi-Fredholm (resp. lower semi-Fredholm) if the range $\mathcal{R}(T)$ is closed and $\operatorname{dim} \mathcal{N}(T)<\infty$ (resp. $\operatorname{codim} \mathcal{R}(T)<\infty)$. If $T$ is either upper or lower semi-Fredholm, then $T$ is called a semi-Fredholm operator. If $T$ is both upper and lower semi-Fredholm, then $T$ is called a Fredholm operator, see ([11]). The upper semi-Fredholm spectrum $\sigma_{u f}(T)$, the lower semi-Fredholm spectrum $\sigma_{l f}(T)$, the spectrum semi-Fredholm $\sigma_{s f}(T)$ and the Fredholm spectrum $\sigma_{f}(T)$ of $T$ are defined by

$$
\begin{gathered}
\sigma_{u f}(T)=\{\lambda \in \mathbb{C}: \lambda-T \text { is not upper semi-Fredholm }\}, \\
\sigma_{l f}(T)=\{\lambda \in \mathbb{C}: \lambda-T \text { is not lower semi-Fredholm }\}, \\
\sigma_{s f}(T)=\{\lambda \in \mathbb{C}: \lambda-T \text { is not semi-Fredholm }\}, \\
\sigma_{f}(T)=\{\lambda \in \mathbb{C}: \lambda-T \text { is not Fredholm }\} .
\end{gathered}
$$

We say that an operator $T \in \mathfrak{C}(X)$ is upper semi-Browder if it is upper semi-Fredholm and has finite ascent. Similarly, $T$ is lower semi-Browder if it is lower semi-Fredholm and has finite descent. An operator $T$ is Browder if it is both lower and upper semi-Browder, see ([13]). The upper semi-Browder spectrum $\sigma_{u b}(T)$, the lower semi-Browder spectrum $\sigma_{l b}(T)$, the spectrum semi-Browder $\sigma_{s b}(T)$ and the Browder spectrum $\sigma_{b}(T)$ of $T$ are defined by

$$
\begin{gathered}
\sigma_{u b}(T)=\{\lambda \in \mathbb{C}: \lambda-T \text { is not upper semi-Browder }\}, \\
\sigma_{l b}(T)=\{\lambda \in \mathbb{C}: \lambda-T \text { is not lower semi-Browder }\}, \\
\sigma_{s b}(T)=\{\lambda \in \mathbb{C}: \lambda-T \text { is not semi-Browder }\}, \\
\sigma_{b}(T)=\{\lambda \in \mathbb{C}: \lambda-T \text { is not Browder }\} .
\end{gathered}
$$

Consider in $X$ the well-posed Cauchy problem

$$
\text { (*) }\left\{\begin{array}{l}
u^{\prime \prime}(t)=A u(t), \quad t \in \mathbb{R} \\
u(0)=u_{0} \\
u^{\prime}(0)=u_{1}
\end{array}\right.
$$

Where $A: X \longrightarrow X$ is a densely defined closed operator with nonempty resolvent set $\rho(A)$. The problem ( $\left.{ }^{\star}\right)$ is (see $[4,10]$ ) well-posed if and only if $A$ generates a strongly continuous cosine operator function $(C(t))_{t \in \mathbb{R}}$, i.e., a family of operators satisfying the following conditions:

1. $C(t+s)+C(t-s)=2 C(t) C(s)$ for all $t, s \in \mathbb{R}$.

2. $C(0)=I$ (the identity operator).

3. $C(t) x$ is strongly continuous with respect to $t$ for any fixed $x \in X$.

There exist some $M \geq 1, \omega \in \mathbb{R}$ such that $\|C(t)\| \leq M e^{\omega t}$ for all $t \geq 0$.

If $(C(t))_{t \in \mathbb{R}}$ is a strongly continuous cosine operator function, then the infinitesimal generating operator $A$ is defined by

$$
\mathcal{D}(A)=\left\{x \in X: \lim _{s \rightarrow 0} \frac{2(C(s) x-x)}{s^{2}} \text { exists }\right\}
$$

and

$$
A x=\lim _{s \rightarrow 0} \frac{2(C(s) x-x)}{s^{2}} .
$$

A solution of problem $(*)$ is given with the help of a strongly continuous cosine operator function by the formula $u(t)=C(t) u_{0}+S(t) u_{1}$ for $t \in \mathbb{R}$, where $S(t)$ is the sine operator function associated with the $(C(t))_{t \in \mathbb{R}}$ and is defined as $S(t) x:=\int_{0}^{t} C(s) x d s, t \in \mathbb{R}, x \in X$. In this work we will use the theory of integration in the sense of Bochner.

If $(C(t))_{t \in \mathbb{R}}$ is a uniformly continuous operator cosine function then there is an $A \in \mathcal{B}(X)$ with $C(t)=\cosh t \sqrt{A}, t \in \mathbb{R}$. We have $A=\lim _{s \rightarrow 0} \frac{2(C(s)-I)}{s^{2}}$ in the uniform operator topology, see [6, Theorem.2.18]. 
For $t \in \mathbb{R}$, the function $f: z \in \mathbb{C} \mapsto \cosh t \sqrt{z}$ defines an entire function. Thus, according to the spectral mapping theorem, we have $\cosh t \sqrt{\sigma_{\star}(A)}=\sigma_{\star}(C(t))$, for all $t \in \mathbb{R}$, with $\sigma_{\star}$ the spectrum corresponding to regularity in the sense of V. Müller [7, Definition 6.1].

In the context of a strongly continuous cosine the following spectral inclusion $\cosh t \sqrt{\sigma(A)} \subseteq$ $\sigma(C(t)), t \in \mathbb{R}$ was obtained by B. Nagy; he also gave an example where the reverse inclusion fails [8] and he showed that $\sigma_{\star}(C(t))=\cosh t \sqrt{\sigma_{\star}(A)}, t \in \mathbb{R}$, with ${ }^{\star} \in\{p, r\}$.

However there are several large classes of generators $A$ for which the spectrum of $C(t)$ can be expressed in terms of $\sigma(A)$, namely $\sigma(C(t))=\overline{\cosh t \sqrt{\sigma(A)}}, t \in \mathbb{R}$, if $A$ is the generator of a uniformly bounded cosine function on a Hilbert space [14] or of a cosine function of normal operators [15].

In this paper, we continue to study the spectral theory of strongly continuous cosine operator function. We investigate the relationships between the different spectra of a strongly continuous cosine operator function and their generators, precisely we prouve that

$$
\cosh t \sqrt{\sigma_{\star}(A)} \cup\{-1,1\} \subseteq \sigma_{\star}(C(t)) \cup\{-1,1\},
$$

where $\sigma_{\star}$ denotes the upper and lower semi-Fredholm, semi-Fredholm, Fredholm, Drazin, upper and lower semi-Browder, semi-Browder, Browder, essential ascent and descent spectra. We show by counter-example that these inclusions are strict in general.

\section{Main results}

The following lemmas are among the most widely used results of this paper.

Lemma 2.1. [8, Lemma. 4] Let $A$ be the generator of the cosine operator function $C$. For $t \in \mathbb{R}$ and $\lambda \in \mathbb{C}$, let

$$
S_{\lambda}(t) x:=\int_{0}^{t} \sinh \lambda(t-s) C(s) x d s, \quad x \in X .
$$

Then $S_{\lambda}(t) \in \mathcal{B}(X)$ is an operator that commutes with $A$, and

$$
\left(A-\lambda^{2}\right) S_{\lambda}(t) x=\lambda(C(t)-\cosh \lambda t) x,
$$

for all $x \in X$

Lemma 2.2. Let $A$ be the generator of a cosine operator function $(C(t))_{t \in \mathbb{R}}$. Then for all $t \neq 0$ and $\lambda \in \mathbb{C}$ with $\lambda t \notin i \pi \mathbb{Z}$, there exist two operators $L_{\lambda}(t), G_{\lambda}(t) \in \mathcal{B}(X)$ such that

$$
\left(A-\lambda^{2}\right) L_{\lambda}(t)+G_{\lambda}(t) S_{\lambda}(t)=I .
$$

Moreover, the operators $L_{\lambda}(t), S_{\lambda}(t), G_{\lambda}(t)$ and $A-\lambda^{2}$ are mutually commuting.

Proof. For all $t \neq 0$ and $\lambda \in \mathbb{C}$ with $\lambda t \notin i \pi \mathbb{Z}$, let $K_{\lambda}(t) x:=\int_{0}^{t} \sinh \lambda(t-s) S_{\lambda}(s) x d s$. It is clear that $K_{\lambda}(t)$ is a

bounded linear operator of $X$. We consider the function $f: s \in[0, t] \longrightarrow \sinh \lambda(t-s) S_{\lambda}(s) x$, then $f$ is Bochner integrable and $f(s) \in \mathcal{D}(A)$ for all $s \in[0, t]$. Moreover, $\left(A-\lambda^{2}\right) f(s)=\lambda \sinh \lambda(t-s)(C(t)-(\cosh \lambda t) I)$ is Bochner 
integrable. From [2, Proposition 1.1.7], $K_{\lambda}(t) x \in \mathcal{D}(A)$ and

$$
\begin{aligned}
\left(A-\lambda^{2}\right) K_{\lambda}(t) x & =\int_{0}^{t} \lambda \sinh \lambda(t-s)(C(s)-\cosh \lambda s) x d s \\
& =\lambda \int_{0}^{t} \lambda \sinh \lambda(t-s) C(s) x d s-\lambda \int_{0}^{t} \sinh \lambda(t-s) \cosh \lambda s x d s \\
& =\lambda S_{\lambda}(t) x-t \lambda \sinh \lambda t x .
\end{aligned}
$$

We put $F_{\lambda}(t):=(-t \lambda \sinh \lambda t)^{-1} K_{\lambda}(t)$ and $G_{\lambda}(t):=(t \sinh \lambda t)^{-1} I$. Then we have $\left(A-\lambda^{2}\right) L_{\lambda}(t)+G_{\lambda}(t) S_{\lambda}(t)=I$. Furthermore, it is clear that the operators $L_{\lambda}(t), S_{\lambda}(t), G_{\lambda}(t)$ and $A-\lambda^{2}$ are mutually commuting.

Lemma 2.3. Let $A$ be the generator of a cosine operator function $(C(t))_{t \in \mathbb{R}}$. Then for all $n \in \mathbb{N}^{\star}, t \neq 0$ and $\lambda \in \mathbb{C}$ with $\lambda t \notin i \pi \mathbb{Z}$, there exist two operators $F_{\lambda, n}(t), H_{\lambda, n}(t) \in \mathcal{B}(X)$ such that,

$$
\left(A-\lambda^{2}\right)^{n} F_{\lambda, n}(t)+H_{\lambda, n}(t) S_{\lambda}^{n}(t)=I .
$$

Moreover, the operators $F_{\lambda, n}(t), H_{\lambda, n}(t), S_{\lambda}^{n}(t)$ and $\left(A-\lambda^{2}\right)^{n}$ are mutually commuting.

Proof. By Lemma 2.2, there exist two operators $L_{\lambda}(t), G_{\lambda}(t) \in \mathcal{B}(X)$ such that

$$
\left(A-\lambda^{2}\right) L_{\lambda}(t)+G_{\lambda}(t) S_{\lambda}(t)=I .
$$

For all $n \geq 1$ and $x \in X$, we have $L_{\lambda}^{n}(t) x \in \mathcal{D}\left(A^{n}\right)$. In fact, the proof is by induction. For $n=1$, from lemma 2.2 $L_{\lambda}(t) x \in \mathcal{D}(A)$. suppose that $L_{\lambda}^{n-1}(t) x \in \mathcal{D}\left(A^{n-1}\right)$, so $L_{\lambda}^{n}(t) x \in \mathcal{D}\left(A^{n-1}\right)$ and

$$
\begin{aligned}
\left(A-\lambda^{2}\right)^{n-1} L_{\lambda}^{n}(t) x & =\left[\left(A-\lambda^{2}\right) L_{\lambda}(t)\right]^{n-1} L_{\lambda}(t) x \\
& =L_{\lambda}(t)\left[\left(A-\lambda^{2}\right) L_{\lambda}(t)\right]^{n-1} x \in \mathcal{D}(A),
\end{aligned}
$$

hence, $L_{\lambda}^{n}(t) x \in \mathcal{D}\left(A^{n}\right)$. Furthermore,

$$
\begin{aligned}
\left(A-\lambda^{2}\right)^{n} L_{\lambda}^{n}(t) & =\left[\left(A-\lambda^{2}\right) L_{\lambda}(t)\right]^{n} \\
& =\left[I-G_{\lambda}(t) S_{\lambda}(t)\right]^{n} \\
& =I-T_{\lambda, n}(t) S_{\lambda}(t),
\end{aligned}
$$

with $T_{\lambda, n}(t)=\sum_{k=1}^{n}(-1)^{k-1}\left(\begin{array}{l}n \\ k\end{array}\right) G_{\lambda}^{k}(t) S_{\lambda}^{k-1}(t)$. So $\left(A-\lambda^{2}\right)^{n} L_{\lambda}^{n}(t)+T_{\lambda, n} S_{\lambda}(t)=I$. Similarly, we have

$$
\begin{aligned}
T_{\lambda, n}^{n}(t) S_{\lambda}^{n}(t) & =\left[I-\left(A-\lambda^{2}\right)^{n} L_{\lambda}^{n}(t)\right]^{n} \\
& =I-\left(A-\lambda^{2}\right)^{n} \sum_{k=1}^{n}(-1)^{k-1}\left(\begin{array}{l}
n \\
k
\end{array}\right)\left(A-\lambda^{2}\right)^{n(k-1)} L_{\lambda}^{n k}(t) .
\end{aligned}
$$

We define $\left.F_{\lambda, n} t\right)=\sum_{k=1}^{n}(-1)^{k-1}\left(\begin{array}{l}n \\ k\end{array}\right)\left(A-\lambda^{2}\right)^{n(k-1)} L_{\lambda}^{n k}(t)$ and $H_{\lambda, n}(t)=T_{\lambda, n}^{n}(t)$. Then $\left(A-\lambda^{2}\right)^{n} F_{\lambda, n}(t)+$ $H_{\lambda, n}(t) S_{\lambda}^{n}(t)=I$. Moreover the operators $\left(A-\lambda^{2}\right)^{n}, F_{\lambda, n}(t), H_{\lambda, n}(t)$ and $S_{\lambda}^{n}(t)$ are pairwise commuting.

Lemma 2.4. Let $A$ be the generator of a cosine operator function $(C(t))_{t \in \mathbb{R}}$. Then for all $q \in \mathbb{N}, t \neq 0$ and $\lambda \in \mathbb{C}$ with $\lambda t \notin i \pi \mathbb{Z}$, if $\mathcal{R}(C(t)-\cosh \lambda t I)^{q}$ is closed, then $\mathcal{R}\left(A-\lambda^{2}\right)^{q}$ is also closed.

Proof. Let $\left(y_{n}\right)_{n \in \mathbb{N}}$ be a sequence in $\mathcal{R}\left(A-\lambda^{2}\right)^{q}$ converging to $y \in X$. Then there is a sequence $\left(x_{n}\right)_{n \in \mathbb{N}}$ of $\mathcal{D}\left(A^{q}\right)$ satisfying $\left(A-\lambda^{2}\right)^{q} x_{n}=y_{n}$. By Lemma 2.2, we obtain

$$
\left(A-\lambda^{2}\right)^{q} F_{\lambda, q}(t) y_{n}+H_{\lambda, q}(t) S_{\lambda}^{q}(t) y_{n}=y_{n} .
$$


Hence, we conclude that

$$
\begin{aligned}
\lambda^{q}(C(t)-\cosh \lambda t)^{q} H_{\lambda, q}^{q}(t) x_{n} & =S_{\lambda}^{q}(t)\left(A-\lambda^{2}\right)^{q} H_{\lambda, q}^{q}(t) x_{n} \\
& =H_{\lambda, q}^{q}(t) S_{\lambda}^{q}(t)\left(A-\lambda^{2}\right)^{q} x_{n} \\
& =H_{\lambda, q}^{q}(t) S_{\lambda}^{q}(t) y_{n} \\
& =y_{n}-\left(A-\lambda^{2}\right)^{q} F_{\lambda, q}(t) y_{n} .
\end{aligned}
$$

Thus, $y_{n}-\left(A-\lambda^{2}\right)^{q} F_{\lambda, q}(t) y_{n} \in \mathcal{R}(C(t)-\cosh \lambda t)^{q}$. Since $\mathcal{R}(C(t)-\cosh \lambda t)^{q}$ is closed and $\left(A-\lambda^{2}\right)^{q} F_{\lambda, q}(t)$ is bounded linear operator, it follows that the sequence $y_{n}-\left(A-\lambda^{2}\right)^{q} F_{\lambda, q}(t) y_{n}$ converges to $y-\left(A-\lambda^{2}\right)^{q} F_{\lambda, q}(t) y$ as $n$ tends to $\infty$ and

$$
y-\left(A-\lambda^{2}\right)^{q} F_{\lambda, q}(t) y \in \mathcal{R}(C(t)-\cosh \lambda t)^{q} \subseteq \mathcal{R}\left(A-\lambda^{2}\right)^{q} .
$$

We obtain $y \in \mathcal{R}\left(A-\lambda^{2}\right)^{q}$, which completes the proof.

Theorem 2.1. Let $A$ be the generator of a cosine operator function $(C(t))_{t \in \mathbb{R}}$. Then for all $t \neq 0$,

$$
\cosh t \sqrt{\sigma_{\star}(A)} \cup\{-1,1\} \subseteq \sigma_{\star}(C(t)) \cup\{-1,1\},
$$

with $* \in\{u f, l f, s f, f\}$.

Proof. Suppose that $C(t)-\cosh t \lambda$ is upper semi-Fredholm, then $\mathcal{N}(C(t)-\cosh \lambda t)$ is finite dimensional and $\mathcal{R}(C(t)-\cosh \lambda t)$ is closed. By Lemma 2.4, we obtain $R\left(A-\lambda^{2}\right)$ is closed. Since $\mathcal{N}\left(A-\lambda^{2}\right) \subseteq \mathcal{N}(C(t)-\cosh \lambda t)$, then $\mathcal{N}\left(A-\lambda^{2}\right)$ is finite dimensional. Therefore $A-\lambda^{2}$ is upper semi-Fredholm.

2. Let $C(t)-\cosh t \lambda$ is lower semi-Fredholm, then $\mathcal{R}(C(t)-\cosh \lambda t)$ is a subspace of $X$ of finite codimension. Since $\mathcal{R}(C(t)-\cosh \lambda t) \subseteq \mathcal{R}\left(A-\lambda^{2}\right)$, then $\mathcal{R}\left(A-\lambda^{2}\right)$ is a subspace of $X$ of finite codimension. Therefore $A-\lambda^{2}$ is lower semi-Fredholm.

3. It is easy by the previous assertions of this theorem.

4. Obvious.

Theorem 2.2. Let $(C(t))_{t \in \mathbb{R}}$ be a strongly continuous cosine function of operators with infinitesimal generator A. Then for all $t \neq 0$,

$$
\cosh t \sqrt{\sigma_{D}(A)} \cup\{-1,1\} \subseteq \sigma_{D}(C(t)) \cup\{-1,1\}
$$

We need the following lemma, which will also be useful later.

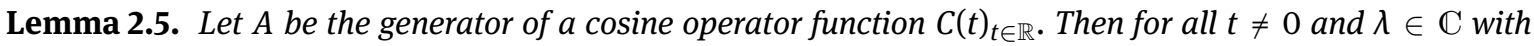
$\lambda t \notin i \pi \mathbb{Z}$,

1. if $d(C(t)-\cosh \lambda t)=n$, then $d\left(A-\lambda^{2}\right) \leq n$.

2. If $a(C(t)-\cosh \lambda t)=n$, then $a\left(A-\lambda^{2}\right) \leq n$.

Proof. If $d(C(t)-\cosh \lambda t)=n$, then $\mathcal{R}(C(t)-\cosh \lambda t)^{n}=\mathcal{R}(C(t)-\cosh \lambda t)^{n+1}$. Let $y \in \mathcal{R}\left(A-\lambda^{2}\right)^{n}$, then there exists $x \in \mathcal{D}\left(A^{n}\right)$ such that $\left(A-\lambda^{2}\right)^{n} x=y$. Then there exists $z \in X$ such that $(C(t)-\cosh \lambda t)^{n} x=$ $(C(t)-\cosh \lambda t)^{n+1} z$. By Lemma 2.2 we have $\left(A-\lambda^{2}\right)^{n} F_{\lambda, n}(t)+H_{\lambda, n}(t) S_{\lambda}^{n}(t)=I$. Thus,

$$
\begin{aligned}
y & =\left(A-\lambda^{2}\right)^{n}\left((\lambda-A)^{n} F_{\lambda, n}(t)+H_{\lambda, n}(t) S_{\lambda}^{n}(t)\right) x, \\
& =\left(A-\lambda^{2}\right)^{2 n} F_{\lambda, n}(t) x+\lambda^{n} H_{\lambda, n}(t)(C(t)-\cosh \lambda t)^{n} x, \\
& =\left(A-\lambda^{2}\right)^{2 n} F_{\lambda, n}(t) x+\lambda^{n} H_{\lambda, n}(t)(C(t)-\cosh \lambda t)^{n+1} z, \\
& =\left(A-\lambda^{2}\right)^{2 n} F_{\lambda, n}(t) x+\lambda^{n} H_{\lambda, n}(t)\left(A-\lambda^{2}\right)^{n+1} S_{\lambda}^{n+1}(t) z, \\
& =\left(A-\lambda^{2}\right)^{n+1}\left(\left(A-\lambda^{2}\right)^{n-1} F_{\lambda, n}(t) x+\lambda^{n} H_{\lambda, n}(t) S_{\lambda}^{n+1}(t) z\right) .
\end{aligned}
$$

So $y \in \mathcal{R}\left(A-\lambda^{2}\right)^{n+1}$, hence $\mathcal{R}\left(A-\lambda^{2}\right)^{n}=\mathcal{R}\left(A-\lambda^{2}\right)^{n+1}$. Finally $d\left(A-\lambda^{2}\right) \leq n$. 
2. If $a(C(t)-\cosh \lambda t)=n$ then $\mathcal{N}(C(t)-\cosh \lambda t)^{n}=\mathcal{N}(C(t)-\cosh \lambda t)^{n+1}$. Let $x \in \mathcal{N}(\lambda-A)^{n+1}$. From Lemma 2.1, $x \in \mathcal{N}(C(t)-\cosh \lambda t)^{n}$. Then,

$$
\begin{aligned}
\left(A-\lambda^{2}\right)^{n} x & =\left(A-\lambda^{2}\right)^{n}\left[\left(A-\lambda^{2}\right)^{n} F_{\lambda, n}(t)+H_{\lambda, n}(t) S_{\lambda}^{n}(t)\right] x, \\
& =\left(A-\lambda^{2}\right)^{2 n} F_{\lambda, n}(t) x+\left(A-\lambda^{2}\right)^{n} H_{\lambda, n}(t) S_{\lambda}^{n}(t) x, \\
& =\left(A-\lambda^{2}\right)^{n+1} F_{\lambda, n}(t) x+\lambda^{n} H_{\lambda, n}(t)(C(t)-\cosh \lambda t)^{n} x, \\
& =\left(A-\lambda^{2}\right)^{n-1} F_{\lambda, n}(t)\left(A-\lambda^{2}\right)^{n+1} x, \\
& =0 .
\end{aligned}
$$

Therefore, $x \in \mathcal{N}\left(A-\lambda^{2}\right)^{n}$ and hence $a\left(A-\lambda^{2}\right) \leq n$.

Theorem 2.1 and Lemma 2.5 imply the following corollary:

Corollary 2.1. Let $(C(t))_{t \in \mathbb{R}}$ be a strongly continuous cosine function of operators with infinitesimal generator A. Then for all $t \neq 0$,

$$
\cosh t \sqrt{\sigma_{\star}(A)} \cup\{-1,1\} \subseteq \sigma_{\star}(C(t)) \cup\{-1,1\},
$$

with * $\in\{u b, l b, s b, b\}$.

Proof of Theorem 2.2. If $C(t)-\cosh \lambda t$ is of invertible Drazin, then the descent and the ascent of $C(t)-$ $\cosh \lambda t$ are finite and equal to $n$. From [7, Theorem.20.4], we have $\mathcal{R}(C(t)-\cosh \lambda t)^{n}$ is closed. By Lemma 2.4, $\mathcal{R}(C(t)-\cosh \lambda t)^{n}$ is closed. According to Lemma 2.5, the descent and the ascent $A-\lambda^{2}$ are finite and from [12, Theorem 2.1], we have $a\left(A-\lambda^{2}\right) \leq d\left(A-\lambda^{2}\right) \leq n$. It is easy to see that $\mathcal{N}\left(A-\lambda^{2}\right)^{n} \cap \mathcal{R}\left(A-\lambda^{2}\right)^{n}=\{0\}$. Indeed if $u \in \mathcal{N}\left(A-\lambda^{2}\right)^{n} \cap \mathcal{R}\left(A-\lambda^{2}\right)^{n}$, then there exists $v \in \mathcal{D}\left(A-\lambda^{2}\right)^{n}$ such that $u=\left(A-\lambda^{2}\right)^{n} v$. And as $u \in \mathcal{N}\left(A-\lambda^{2}\right)^{n}$, we see that $v \in \mathcal{N}\left(A-\lambda^{2}\right)^{2 n}=\mathcal{N}\left(A-\lambda^{2}\right)^{n}$ and therefore $u=\left(A-\lambda^{2}\right)^{n} v=0$. Let us show that $X=\mathcal{R}\left(A-\lambda^{2}\right)^{n}+\mathcal{N}\left(A-\lambda^{2}\right)^{n}$. As $d\left(A-\lambda^{2}\right) \leq n$, then in particular we have $\mathcal{R}\left(A-\lambda^{2}\right)^{n}=\mathcal{R}\left(A-\lambda^{2}\right)^{2 n}$. So if $u \in \mathcal{D}\left(A-\lambda^{2}\right)^{n}$, then $\left(A-\lambda^{2}\right)^{n} u \in \mathcal{R}\left(A-\lambda^{2}\right)^{n}=\mathcal{R}\left(A-\lambda^{2}\right)^{2 n}$. So there exists $v \in \mathcal{D}\left(A-\lambda^{2}\right)^{2 n}$ such that $A^{n} u=\left(A-\lambda^{2}\right)^{2 n} v$. Hence $u-\left(A-\lambda^{2}\right)^{n} v \in \mathcal{N}\left(A-\lambda^{2}\right)^{n}$. Consequently $u \in \mathcal{R}\left(A-\lambda^{2}\right)^{n}+\mathcal{N}\left(A-\lambda^{2}\right)^{n}$ and therefore $\mathcal{D}\left(A-\lambda^{2}\right)^{n} \subseteq \mathcal{R}\left(A-\lambda^{2}\right)^{n}+\mathcal{N}\left(A-\lambda^{2}\right)^{n}$. According [2, Theorem 3.14.17], $A$ generates a strongly continuous semigroup and from [3, 1.8 Proposition], $\mathcal{D}\left(A^{n}\right)$ is dense in $X$, since $\mathcal{R}\left(A-\lambda^{2}\right)^{n}+\mathcal{N}\left(A-\lambda^{2}\right)^{n}$ is closed, then $X=\mathcal{R}\left(A-\lambda^{2}\right)^{n}+\mathcal{N}\left(A-\lambda^{2}\right)^{n}$. From [1, Theorem 1.35], we have $A-\lambda^{2}$ is of invertible Drazin, which finishes the proof.

Lemma 2.6. Let $(C(t))_{t \in \mathbb{R}}$ be a strongly continuous cosine function of operators with infinitesimal generator $A$. Then for all $t \neq 0$ and $\lambda \in \mathbb{C}$ with $\lambda t \notin i \pi \mathbb{Z}$,

1. if $d_{e}(C(t)-\cosh \lambda t)=n$, then $d_{e}\left(A-\lambda^{2}\right) \leq n$.

2. If $a_{e}(C(t)-\cosh \lambda t)=n$, then $a_{e}\left(A-\lambda^{2}\right) \leq n$.

Proof. Suppose that $C(t)-\cosh \lambda t$ has finite essential descent, then there exists $n \in \mathbb{N}$ such that $\mathcal{R}(C(t)-$ $\cosh \lambda t)^{n} / \mathcal{R}(C(t)-\cosh \lambda t)^{n+1}$ is finite dimensional. Let

$$
\phi: \mathcal{R}\left(A-\lambda^{2}\right)^{n} \longrightarrow \mathcal{R}(C(t)-\cosh \lambda t)^{n} / \mathcal{R}(C(t)-\cosh \lambda t)^{n+1}
$$

the mapping defined by

$$
\phi\left((\lambda-A)^{n} x\right)=(C(t)-\cosh \lambda t)^{n} x+\mathcal{R}(C(t)-\cosh \lambda t)^{n+1} .
$$

Thus, by isomorphism Theorem, we obtain $\mathcal{R}\left(A-\lambda^{2}\right)^{n} / \mathcal{N}(\phi)$ is isomorphic to $\mathcal{R}(C(t)$ $\cosh \lambda t)^{n} / R(C(t)-\cosh \lambda t)^{n+1}$. Therefore $\mathcal{R}(\lambda-A)^{n} / N(\phi)$ is finite dimensional. Since $\mathcal{N}(\phi) \subseteq$ $\mathcal{R}(C(t)-\cosh \lambda t)^{n+1} \subseteq \mathcal{R}\left(A-\lambda^{2}\right)^{n+1}$, then $\mathcal{R}(\lambda-A)^{n} / \mathcal{R}(\lambda-A)^{n+1} \subseteq \mathcal{R}(\lambda-A)^{n} / \mathcal{N}(\phi)$. Finally, $A-\lambda^{2}$ has finite essential descent. 
2. Suppose that $C(t)-\cosh \lambda t$ has finite essential ascent. Then $\mathcal{N}(C(t)-\cosh \lambda t)^{n+1} / \mathcal{N}(C(t)-\cosh \lambda t)^{n}$ is finite dimensional. Let

$$
\psi: \mathcal{N}(\lambda-A)^{n+1} \longrightarrow \mathcal{N}(C(t)-\cosh \lambda t)^{n+1} / \mathcal{N}(C(t)-\cosh \lambda t)^{n}
$$

the mapping defined by

$$
\psi(x)=x+\mathcal{N}(C(t)-\cosh \lambda t)^{n} .
$$

By isomorphism Theorem, $\mathcal{N}(\lambda-A)^{n+1} / N(\psi)$ is isomorphic to $\mathcal{R}(\psi)$, since $\mathcal{R}(\psi) \subseteq \mathcal{N}(C(t)-\cosh \lambda t)^{n+1} / \mathcal{N}(C(t)-$ $\cosh \lambda t)^{n}$, Then $\mathcal{N}(\lambda-A)^{n+1} / \mathcal{N}(\psi)$ is finite dimensional. From lemma 2.3, we have,

$$
\mathcal{N}(\psi) \subseteq \mathcal{N}(\lambda-A)^{n+} \cap \mathcal{N}(C(t)-\cosh \lambda t)^{n} \subseteq \mathcal{N}(\lambda-A)^{n},
$$

hence,

$$
\mathcal{N}(\lambda-A)^{n+1} / \mathcal{N}(\lambda-A)^{n} \subseteq \mathcal{N}(\lambda-A)^{n+1} / \mathcal{N}(\psi) .
$$

Finally, $\left.\mathcal{N}(\lambda-A)^{n+1} / \mathcal{N}(\lambda-A)^{n}\right)$ is finite dimensional.

Theorem 2.3. Let $C(t)_{t \in \mathbb{R}}$ be a strongly continuous cosine function of operators with infinitesimal generator $A$. Then for all $t \neq 0$,

$$
\cosh t \sqrt{\sigma_{\star}(A)} \cup\{-1,1\} \subseteq \sigma_{\star}(C(t)) \cup\{-1,1\},
$$

with $\sigma_{\star} \in\left\{\sigma_{l d}^{e}, \sigma_{r d}^{e}\right\}$.

Proof. The conclusion follows from Lemma 2.4 and Lemma 2.6

Remark 2.1. Let $X$ be the complex $l_{2}$ space, and for $\left(z_{n}\right)_{n \in \mathbb{N}} \in l_{2}, s \in \mathbb{R}$ put $C(s)\left(z_{n}\right)_{n}=\left(\cos (n s) z_{n}\right)_{n}$. Then $A\left(z_{n}\right)_{n}=\left(-n^{2} z_{n}\right)_{n}$ with $\mathcal{D}(A)=\left\{\left(z_{n}\right)_{n} \in l_{2}: \sum_{n=1}^{\infty} n^{4}\left|z_{n}\right|^{2}<\infty\right\}$ and $\sigma(A)=\sigma_{p}(A)=\left\{-n^{2}: n \in \mathbb{N}^{\star}\right\}$.

Then $\cosh \sqrt{\sigma_{\star}(A)}$ is countable, with $\sigma_{\star} \in\left\{\sigma_{u f}, \sigma_{l f}, \sigma_{s f}, \sigma_{f}, \sigma_{D}, \sigma_{u b}, \sigma_{l b}, \sigma_{s b}, \sigma_{b}, \sigma_{l d}^{e}, \sigma_{r d}^{e}\right\}$. From [8], $\sigma(C(1))$ contains the set $[-1,1] \backslash\left\{\cos n: n \in \mathbb{N}^{\star}\right\}$ which is uncountable set. Then $\sigma(C(1))$ is uncountable. By [9, Corollary 2.10], we have $\sigma_{\star}(C(1))$ is uncountable, with $\sigma_{\star} \in\left\{\sigma_{u f}, \sigma_{l f}, \sigma_{s f}, \sigma_{f}, \sigma_{D}, \sigma_{u b}, \sigma_{l b}, \sigma_{s b}, \sigma_{b}, \sigma_{l d}^{e}, \sigma_{r d}^{e}\right\}$. This shows that all of the above inclusions are strict.

Question 2.1. Under what conditions were equal in the previous incusions?

\section{References}

[1] P. Aiena, Fredholm and local spectral theory II, Lecture Notes in Mathematics, 2235, Springer, Cham, 2018.

[2] W. Arendt, C. J. K. Batty, M. Hieber, and F. Neubrander, Vector-valued Laplace Transforms and Cauchy Problems, Monographs in Mathematics, 96, Birkhäuser-Springer, Basel, 2011.

[3] K.J. Engel, R. Nagel, One-Parameter Semigroups for Linear Evolution Equations, Graduate Texts in Mathematics, vol. 194. Springer, New York (2000)

[4] H. O. Fattorini, Ordinary differential equations in linear topological space, II, J. Differential Equations 6 (1969), 50-70.

[5] Z. Garbouj and H. Skhiri, Essential ascent of closed operator and some decomposition theorems, Commun. Math. Anal. 16(2) (2014), 19-47.

[6] D. Lutz, Strongly continuous operator cosine functions, Functional Analysis (Dubrovnik, 1981). (Lecture Notes in Math., Vol. 948), Springer, Berlin-New York, 1982, 73-97.

[7] V. Müller, Spectral Theory of Linear Operators and Spectral Systems in Banach Algebras, 2nd edition. Oper. Theory Advances and Applications, vol. 139 (2007).

[8] B. Nagy, On cosine operator functions in Banach spaces, Acta Sci. Math. Szeged 36 (1974), 281-290.

[9] A. Tajmouati, M. Amouchb, M. Karmouni Symmetric Difference Between Pseudo B-Fredholm Spectrum and Spectra Originated from Fredholm Theory, Filomat 31(16)(2017), 5057-5064.

[10] C. C. Travis and G. F. Webb, Cosine families and abstract nonlinear second order differential equations, Acta Math. Acad. Sci. Hungar. 32 (1978),75-96. 
[11] V. Williams, Closed Fredholm and Semi-Fredholm Operators, Essential Spectra and Perturbations, J. Functional Anal. 20 (1975), 1-25.

[12] M. Zuhair, Nashed and Yagu Zhao, The Drazin Inverse for Singular Evolution Equations and Partial Differential Operators, WSSIAA 1 (1992), 441-456

[13] S.Č. Živković-Zlatanović, M. D. Cvetković and D. S. Djordjević, On Closed Upper and Lower Semi-Browder Operators, Mediterr. J. Math. 12 (3) (2014), 1033-1045.

[14] H. O. Fattorini, Uniformly bounded cosine functions in Hilbert spaces, Indiana Univ. Math. J. 20 (1970), 411-425.

[15] S. Kurepa, cosine functkmal equation in Hilbert space, Canad. J. Math., 12 (1960), 45-49. 